RESEARCH ARTICLE

\title{
Endoscopic Vesicoureteral Reflux Treatment Outcomes in Patients with Voiding Dysfunction
}

\author{
Elif Altınay Kırlı, Çağatay Doğan, Mehmet Hamza Gültekin, Zübeyr Talat and Bülent Önal*
}

Istanbul University-Cerrahpaşa Cerrahpaşa School of Medicine, Departmant of Urology, Turkey

*Corresponding author: Bülent Önal, MD, Professor, Department of Urology, Cerrahpaşa Faculty of Medicine, Istanbul University, Turkey, Tel: +90-543-7955564; +90-212-4143000/21200

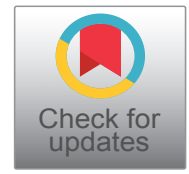

\begin{abstract}
Objective: Voiding dysfunction (VD) increases the frequency of vesicoureteral reflux (VUR). Subureteric injection is an effective method for the treatment; however, the presence of VD may affect the success rates. This study evaluated the results of single-session endoscopic treatment in patients admitting with VD symptoms who were diagnosed with VUR, and the effect of compliance to VD treatment on these results.
\end{abstract}

Materials and method: The data regarding patients who were being followed up for non-neurogenic VD diagnosis and who underwent subureteric injection or treated conservatively was reviewed retrospectively in terms of follow up information and surgical outcomes. Endoscopic treatment was performed by applying dextranomer/ hyaluronic acid copolymer via submucosal route. Symptomatic and radiological assessments of the patients on month 3 were reviewed.

Results: The data of 45 patients (67 renal unites (RU)) were assessed. 24 of them treated conservatively 21 of them underwent endoscopic subureteric injection (STING). STING was performed for Grade 1 VUR in $3 \mathrm{RU}$, grade 2 in $12 \mathrm{RU}$, grade 3 in $16 \mathrm{RU}$, grade 4 in $2 \mathrm{RU}$, and grade 5 in 2 $\mathrm{RU}$. The success rate after subureteric injection treatment was found to be $68 \%$. VD treatment incompliance rate was detected to be $31 \%$, and infection rate during follow-up after injection to be $24 \%$.

Conclusion: Providing regular treatment for voiding dysfunction for at least 6 months before the endoscopic injection treatment for VUR accompanied by VD is important for increasing the success rates. That being said, the success rate in this group might be lower than expected despite proper treatment for bladder.

\section{Keywords}

Vesicoureteral reflux, Voiding dysfunction, Subureteric injection

\section{Introduction}

Vesicoureteral reflux (VUR) is the most frequent urinary system anomaly of the pediatric population with $1 \%$ incidence $[1,2]$. The main etiological cause is the anatomically inadequate anti-reflux mechanism.

Non-neuropathic lower urinary tract dysfunction (LUTD) is based purely on the fact that any neurologic lesions that can affect the lower urinary system can be identified [3]. In patients who also have voiding dysfunction (VD), especially who have impaired bladder compliance; VUR may develop due to impaired bladder dynamic [4-6].

Filling-phase and voiding phase dysfunctions are the two main groups of LUTD. Detrusor can be over active (DOA) as in over active bladder (OAB) or underactive as in underactive bladder. Detrusor underactivity (DUA) denotes voiding contraction of reduced strength resulting prolonged bladder emptying or failure to achieve complete emptying. Habitual postponing the micturition is also in this group. Detrusor-sphincter interference is the main problem of voiding phase. This condition can be a transitional phases of a complex sequence due to fact that should not be viewed as distinct entities $[3,7,8]$.

The common problem in patients with VUR and VD is the renal injury developed as a result of urinary tract infections (UTIs) [9]. The coexistence of two pathologies may cause increase in the risk of renal failure. Therefore, VD investigation is an important part of the diagnosis process in children diagnosed with VUR.

Citation: Kırlı EA, Doğan C, Gültekin MH, Talat Z, Önal B (2019) Endoscopic Vesicoureteral Reflux Treatment Outcomes in Patients with Voiding Dysfunction. Int Arch Urol Complic 5:052. doi. org/10.23937/2469-5742/1510052

Accepted: January 26, 2019: Published: January 28, 2019

Copyright: (C) 2019 Kırlı EA, et al. This is an open-access article distributed under the terms of the Creative Commons Attribution License, which permits unrestricted use, distribution, and reproduction in any medium, provided the original author and source are credited. 
Spontaneous resolution may occur in low-grade VUR especially detected in early childhood. That being said, in cases in which urinary tract infection cannot be controlled and scarring occurs, the preferred treatment is surgery irrespective of VUR grade [1]. Endoscopic subureteric injection (STING) in VUR treatment is a minimally invasive and well-tolerated method, which provides a cure rate similar to open surgery. Today, it is preferred as primary surgical approach for VUR [10-12]. Also, endoscopic approach in the patients with neuropathic bladder who may also have many comorbidities has been described as a preferable method compared to open surgical approaches [10-13].

This study mainly evaluated the efficacy of singlesession STING in a patient group detected to have coexisting VD and VUR, and the effect of compliance to VD treatment on success rates. Also provides information about the follow-up of patients followed by conservatively.

\section{Materials and Method}

Medical records of 150 patients (216 renal unit (RU)) who diagnosed as VUR in the past 10 years were evaluated retrospectively. Patients who were treated due to VUR accompanied by VD and evaluated with DMSA and voiding cystourethrography examination at the time of diagnosis and at least one VCUG examination during follow-up or post-operative period were accepted to study. $67 \mathrm{RU}$ of a total of 45 patients with complete medical records, were assessed retrospectively for affect of medical treatment and urotherapy compliance on clinical findings and follow up period characteristics.

VUR grades were assessed radiologically by performing voiding cystourethrography before and after treatment [14]. All patients were assessed for VD before the endoscopic intervention using voiding diary and if clinically indicated, urodynamics. Voiding dysfunction symptom score was used for quantitative systematic review of the voiding symptoms and also used for follow-up [15].

DUA diagnoses were made using urodynamics, and $O A B$ diagnosis based on symptoms and urodynamics findings. Oxybutynin $(0.1-0.3 \mathrm{mg} / \mathrm{kg}, 3$ times daily) was initiated for antimuscarinic treatment in patients diagnosed with DOA or OAB.

Post-void residual urine was evaluated according to age-based criteria of International Children's Continence Society and clean intermittent catheterization was recommended in DUA patients with post-void residual urine [8]. Patients were assessed for constipation and urinary tract infection in their initial and follow-up visits.

All patients were introduced for standard urotherapy. Educational session at the standard urotherapy consist of informative session (for anatomy and function of urinary tract, abdominal and pelvic muscles), habitual regulations as fluid intake, regular voiding and dietary regulation for preventing constipation. Optimal voiding position was demonstrated with the child in front of a mirror. Micturition was supervised and corrections made until achieving patient's cooperation [8].

In addition to standard urotherapy, antibiotherapy was initiated at suppressive dose in patients with UTI that was proved by urinary culture (trimethoprim (1-2 $\mathrm{mg} / \mathrm{kg}$ )- Sulfamethoxazole (10-15 mg/kg) or nitrofurantoin (1 $\mathrm{mg} / \mathrm{kg}$ ) according to urinary culture features), and laxative treatment was initiated for patients with the complaint of constipation. The continuity of antimuscarinic treatment, antibiotic suppression and standard urotherapy for 6 months was accepted as compliance to medical treatment.

Dimercaptosuccinic acid static scintigraphy (DMSA) scanning was performed as a initial scan for renal function and scarring. Additionally, in the case of recurrent UTI or pyelonephritis under the antibiotic suppression is the other indication of DMSA scans for evaluating new scar formation.

STING was performed in all RU that require surgical management. STING indication for low-grade reflux is UTI persisting under suppressive therapy and the detection of scarring in dimercaptosuccinic acid static scintigraphy (DMSA) in renal unit (RU) at the time of diagnosis.

Single-session subureteric injection treatment is performed by applying classic STING technique, which was described by O'Donnell and Puri. Zero lens cystoscope was used to identify ureterovesical junction (UVJ) and $5 \mathrm{Fr}$ needle was inserted at 6 o'clock position. After entering the mucosa 2-3 mm distal to UVJ, needle was advanced to submucosal plane for a distance of 4-5 $\mathrm{mm}$. An average of 0.5 cc of Dextranomer/hyaluronic acid copolymer injection was performed until the mound becomes apparent [16].

Follow-up for the patients after the procedure was performed with routine outpatient clinic visits, and radiologically, using USG at month 1 , and USG and VCUG at month 6. Complete regression or downgrading of reflux in VCUG was considered as success and persisting or increasing reflux grade during follow-up was considered as failure. Statistical evaluation was performed using Wilcoxon Signed Rank test.

\section{Results}

67 RU belong to forty-five patients ( 34 females, 11 males) were evaluated. Twenty-four patients (32 RU) were treated conservatively and 21 patients (35 RU) had STING procedure.

First presenting complaints were detected to be UTI $(n=23,51 \%)$, incontinence $(n=15,33 \%)$, previously 
Table 1: Demographic features of patients according to treatment approach.

\begin{tabular}{|l|l|l|l|}
\hline & & \multicolumn{2}{l|}{ Treatment Approach (n, \%) } \\
\hline & & $\begin{array}{l}\text { Conservative } \\
24(53)\end{array}$ & $\begin{array}{l}\text { Surgical Intervention } \\
21(47)\end{array}$ \\
\hline Age (year) & & $7.2 \pm 3.6$ & $7.9 \pm 8.2$ \\
\hline Gender & Male & $3(12)$ & $8(38)$ \\
\hline & Female & $21(88)$ & $13(62)$ \\
\hline Complaint & UTI & $15(62)$ & $8(38)$ \\
\hline & VUR & $1(4)$ & $3(14)$ \\
\hline & Abdominal pain & $0(0)$ & $3(14)$ \\
\hline Diagnose & Incontinence & $8(34)$ & $7(34)$ \\
\hline & Over active bladder & $23(95)$ & $18(86)$ \\
\hline & Detrusor underactivity & $1(5)$ & $3(14)$ \\
\hline
\end{tabular}

Table 2: Evaluation of RU according to treatment approach and compliance to urotherapy and medical treatment.

\begin{tabular}{|l|l|l|l|l|}
\hline & \multicolumn{4}{|c|}{ Treatment Approach } \\
\hline Treatment Response & Conservative Treatment & Surgical Intervention \\
\hline & Compatible & Incompatible & Compatible & Incompatible \\
\hline Resolution & $18(56)$ & $3(9)$ & $15(43)$ & $1(3)$ \\
\hline Downgrading & $5(16)$ & $2(7)$ & $6(17)$ & $2(5)$ \\
\hline Stable & $1(3)$ & $3(9)$ & $3(9)$ & $8(23)$ \\
\hline Total & $24(75)$ & $8(25)$ & $24(69)$ & $11(31)$ \\
\hline
\end{tabular}

detected VUR ( $n=4,9 \%)$ and abdominal pain $(n=3$, $7 \%)$.

After the initial evaluation of the patients, the presence of OAB ( $n=41,91 \%)$ symptoms and findings, DUA ( $n=4,9 \%$ ), and the coexistence of VUR were detected. Demographic features of patients according to treatment approach were summarized in Table 1.

Grade 1 VUR in 8 (25\%), grade 2 in 13 (41\%), grade 3 in $9(28 \%)$ and grade 4 in $2(6 \%)$ were detected in pretreatment VCUG examination of patients who managed conservatively. VUR was detected in left in 13 (54\%) right in $3(13 \%)$ and bilateral in $8(33 \%)$ patients. Grade 1 VUR in 3 (9\%), grade 2 in 12 (34\%), grade 3 in $16(45 \%)$ and grade 4 in $2(6 \%)$ grade 5 in $2(6 \%)$ were detected in pre-treatment VCUG examination of patients who managed surgically. VUR was detected in left in 7 (33\%) and bilateral in 14 (66\%) patients.

DMSA examination at the time of diagnosis in patients who required surgical management ( $n=32 \mathrm{RU}$ ) showed function below $40 \%$ in $7 \mathrm{RU}(22 \%)$ and scarring in $12 \mathrm{RU}(38 \%)$. Renal scarring was detected in 4 bilateral VUR patients (3 patients affected bilateral). Patient treated conservatively have normal renal functions without any scar formation in DMSA examination.

When the patients were evaluated according to the standard urotherapy and medical treatment compliance, it was seen that the compliance of the patients treated with conservative treatment was 75\%. Decrease of reflux grade in post treatment VCUG is statistically significant with a success rate of $72 \%(p=0.001)$. The rate of success in patients who do not comply with treatment is $16 \%$. There was no significant decrease in VUR grade ( $p=0.0039)$ during follow up.

The compliance of the patients treated with STING was $69 \%$. Decrease of reflux grade in post treatment VCUG is statistically significant and success rate $s$ is $60 \%$ in these patients $(p=0.001)$. The rate of success in patients who do not comply with treatment is $31 \%$. There was no significant decrease in VUR grade $(p=$ 0.063) after injection. Evaluation of RUs according to treatment approach and compliance to standard urotherapy and medical treatment were summarized in Table 2.

During follow-up, it was detected that $11 \%$ ( $\mathrm{n}=$ $5,9 \mathrm{RU}$ ) of the patients had recurrent urinary tract infection with fever. All these patients were previously underwent STING due to recurrent UTI in conservative treatment. Pyuria detected in urine analysis and urine cultures were positive for bacteria. Two patients diagnosed as pyelonephritis. Three (15\%) of the patients with recurrent UTI had new scarring in DMSA. Nine RU belongs to these patients underwent open surgical intervention due to repeating UTI episodes. Although the grade of VUR in this RU decreased after reimplantation surgery recurrent UTI was detected in a patient (1 RU, 12\%). Grade of VUR decrease in 4 (44\%), resolve in $4 \mathrm{RU}(44 \%)$ after reimplantation and UTI did not occur in these patients under suppression.

Other patients who underwent STING or following conservatively did not have documented UTI in follow-up.

Nineteen of conservatively treated patients had constipation at the time of diagnosis. Constipation improved in 12 in compatible, 3 in incompatible patients. Seven of surgically treated patients had constipation at the time of diagnosis. Constipation improved in 6 in compatible, 1 in incompatible patient.

Urinary incontinence was detected as presenting symptom in 15 patients and none of these patients has VUR more then grade 3. Symptom completely resolves 
in 11 patients after standard urotherapy and medical management.

No injection material migration or an obstructive complication was detected in patients who underwent endoscopic treatment or open surgery.

\section{Discussion}

VUR resolution rate following single injection for grade 1 and 2 was $78 \%, 72 \%$ for grade 3,63\% for grade 4 and $51 \%$ for grade 5 . The total success rate with one and multiple injection was $85 \%$. The success rate was reported as $74 \%$ for normal, $62 \%$ for neuropathic bladder [17]. This study detected a success rate of $68 \%$ for endoscopic treatment in the patient group with coexisting VD and VUR. While this rate is lower compared to the patients without VD who underwent endoscopic treatment, it is consistent with the finding of low success rates after neurogenic or non-neurogenic bladder interventions as reported in larger series $[10,11,17]$.

Läckgren, et al. have reported that repeating endoscopic treatment in coexisting bladder dysfunction and VUR provides a success rate of $83 \%$ [14]. The fact that treatment outcomes of all of the study patients were evaluated after single-session injection in our study is a negative factor on our success rate, and also, most of the study patients had grade 3 or higher reflux grade. The reason for not repeating STING is the occurrence of frequent UTI episodes in patients who were decided to undergo open surgery and the detection of new scarring during follow-up. One of the main goals in VUR treatment is preventing UTI, which may lead to renal failure $[1,9]$. In the same study, it has been stated that bladder dysfunction resolved in $59 \%$ of the patients and urinary tract infection was not observed again in $83 \%$ of the patients [14]. While we do not think that endoscopic treatment has effects on bladder functions, we believe that previously administered anticholinergic treatment has effect on this outcome. In this respect, the success rate in study subjects is higher that the resolution rate of VUR. The findings of regression of VD complaints and the decreasing incidence of urinary tract infections are consistent with this study.

It is known that UTI and reflux grade decreases using proper bladder treatment in patients who are being followed-up for VD. The preparation of proper bladder background for urinary tract surgeries to be performed is a factor which increasing the success rates $[1,17]$. In conservatively treated patient's success rate is higher, who were compatible to urotherapy and regular use of medical treatment. When we evaluated the presenting complaints of the patients, we detected that symptoms suggesting VD such as incontinence are present in a low number of patients, and signs which develop as a result of combined UTI and VUR even at low grades are in the forefront. Suppression of overactivity and UTI may lead to resolve of urinary incontinence. The fact that spontaneous resolution was not detected as at high rates as expected was also a warning sign for VD. The positive correlation between constipation and VD was clearly reported in the literature [18]. We detected that this pathology which may cause VD in a low number of patients at admittance was questioned and treated at low rates. Therefore, we believe that in case a UTI which cannot be controlled using prophylaxis especially in bilateral and low-grade VUR is detected, performing a detailed assessment for VD and constipation would affect the treatment period positively. In this study, proper medical (prophylactic antibiotic, antimuscarinic) and behavioral treatment (standard urotherapy) was initiated in all patients before endoscopic surgery. The success rate was found to be high in the patients, which complied with treatment. This can be explained by the fact that the bladder treatment prepared a proper background for endoscopic surgery. Therefore, it should be remembered that the success rates in patients with VD may not be at the expected level. Urinary diversion could be a temporarily option till improving patient compliance and bladder condition. Surgical cure may be preferred in patients who had or would have failure with endoscopic treatment.

As for complications, in their study, Capozza, et al. have reported that most of the patients with implant migration and who were considered to have treatment failure are being followed-up for VD diagnosis [11]. In our study, obstructive complications was not detected in patients who underwent endoscopic treatment or open surgery.

\section{Conclusion}

Management of VD reduces the requirement of surgical intervention by reducing the rate of UTI and leads to downgrading or complete resolution of VUR. Treatment for VD before the endoscopic treatment may provide increase in success rates Therefore, in VUR treatment, patients should be informed for importance of compliance to medical treatment for VD, and if endoscopic injection treatment is being planned, a regular treatment must be provided for at least 6 months before the procedure. It should be remembered that success rates may be low despite proper VD treatment, and surgical approaches should be in the forefront if necessary.

\section{Financial Disclosure}

The authors declared that this study received no financial support.

\section{Ethics Committee Approval}

This study was approved by Istanbul University Ethical Committee.

All authors equally contributed to the manuscript.

The authors have nothing to disclosure. 


\section{References}

1. Tekgül S, Doğan HS, Hoebeke P, Kocvara R, Nijman JM, et al. (2016) Vesicoureteric reflux. EAU Guidelines on Paediatric Urology, European Association of Urology, 5156.

2. Jacobson SH, Hansson S, Jakobsson B (1999) Vesicoureteric reflux: Occurrence and long-term risks. Acta Paediatr Suppl 88: 22-30

3. Tekgül S, Doğan HS, Hoebeke P, Kocvara R, Nijman JM, et al. (2016) Day-time lower urinary tract conditions. EAU Guidelines on Paediatric Urology, European Association of Urology, 37-39.

4. Koff SA, Lapides J, Piazza DH (1979) Association of urinary tract infection and reflux with uninhibited bladder contractions and voluntary sphincteric obstruction. J Urol 122: 373-376.

5. Nasrallah PF, Simon JW (1984) Reflux and voiding abnormalities in children. Urology 24: 243-245.

6. Homsy YL, Nsouli I, Hamburger B, Laberge I, Schick E (1985) Effects of oxybutynin on vesicoureteral reflux in children. J Urol 134: 1168-1171.

7. Van Gool JD, Hjalmas K, Tamminen-Mobius T, Olbing $\mathrm{H}$ (1992) Historical clues to the complex of dysfunctional voiding, urinary tract infection and vesicoureteral reflux. The international reflux study in children. J Urol 148: 16991702.

8. Austin PF, Bauer SB, Bower W, Chase J, Franco I, et al. (2016) The standardization of Terminology of lower urinary tract function in children and adolescent: Update report from the standardization committee of International children's continence society. Neurourol and Urodyn 35: 471-481.

9. Mattoo TK (2011) Vesicoureteral reflux and reflux nephropathy. Adv Chronic Kidney Dis 18: 348-354.
10. Van Batavia JP, Nees SN, Fast AM, Combs AJ, Glassberg $\mathrm{KI}$ (2014) Outcomes of vesicoureteral reflux in children with non-neurogenic lower urinary tract dysfunction treated with dextranomer/hyaluronic acid copolymer (Deflux). J Pediatr Urol 10: 482-487.

11. Capozza N, Caione P, De Gennaro M, Nappo S, Patricolo M (1995) Endoscopic treatment of vesico-ureteric reflux and urinary incontinence: Technical problems in the paediatric patient. Br J Urol 75: 538-542.

12. Polackwich AS, Skoog SJ, Austin JC (2012) Long-term followup after endoscopic treatment of vesicoureteral reflux with dextranomer/hyaluronic acid copolymer in patients with neurogenic bladder. J Urol 188: 1511-1515.

13. Routh JC, Vandersteen DR, Pfefferle H, Wolpert JJ, Reinberg Y (2006) Single center experience with endoscopic management of vesicoureteral reflux in children. J Urol 175: 1889-1892.

14. Lebowitz RL, olbing $H$, Parkkulainen KV, Smellie JM, Tamminen-Möbius TE (1985) International system of radiographic grading of vesicoureteric reflux. International reflux study in children. Pediatr Radiol 15: 105-109.

15. Akbal C, Genc Y, Burgu B, Ozden E, Tekgul S (2005) Dysfunctional voiding and incontinence scoring system: Qualitative evaluation of incontinence symptoms in pediatric population. J Urol 173: 969-973.

16. O'donnell B, Puri P (1986) Endoscopic correction of primary vesicoureteral reflux. BR J Urol 58: 601-604.

17. Elder JS, Diaz M, Caldamone AA, Cendron M, Greenfield $S$, et al. (2006) Endoscopic therapy for vesicoureteral reflux: A meta-analysis. I. Reflux resolution and urinary tract infection. J Urol 175: 716-722.

18. Chung KL, Chao NS, Liu CS, Tang PM, Liu KK, et al. (2014) Abnormal voiding parameters in children with severe idiopathic constipation. Pediatr Surg Int 30: 747-749. 